different patterns of faunal regions during the Mesozoic, and that these correlated well with current ideas of the varying patterns of sea barriers due partly to continental drift and partly to marine transgressions.

An elegant synthesis of electromyographic recording and of X-ray ciné film was used by K. Hiiemae (Guy's Hospital Medical School) to analyse the interrelationships between jaw movement and muscle contraction in the opossum.

\section{When particles hit nuclei}

\author{
from D. J. Miller
}

How are excited states transmitted through nuclei? For some years it has been clear that we do not know the answer to this question. Surprising results were seen first in coherent production processes, where a beam particle is excited into a low-mass cluster of fast particles, without exciting or breaking the nuclear target. It seems that the fast cluster as a whole has just the same chance of interacting again, on its way out of the nucleus where it is produced, as would a single particle. This applies to clusters of two, three or five fast particles, produced by beams of gamma rays, mesons or nucleons on nuclear targets from deuterium to uranium.

Recent results from the Fermilab (Fermi National Accelerator Laboratory) near Chicago have extended our knowledge of these effects, though there is still no clear explanation. A topical meeting on high energy collisions involving nuclei was held in September at the International Centre for Theoretical Physics in Trieste. New results were reported on a whole range of related topics, including data on the multiplicity of secondary particles in particle-nucleus collisions. Nuclear emulsion workers have shown that the multiplicity of fast charged tracks produced in $200 \mathrm{GeV}$ proton-nucleus collisions is only a modest factor larger than the multiplicity in proton-proton collisions at the same energy. For the mixture of nuclei in photographic emulsion, including a significant amount of bromine and silver, the factor is 1.7 , and it has this value from about $70 \mathrm{GeV}$ all the way up to 400 GeV. Busza's group at the Massachusetts Institute of Technology, with a simple Cerenkov counter experiment at Fermilab, has shown that the multiplicity of fast charged particles increases slowly with the atomic mass number $A$ of the nuclide in the target, though the multiplicity in the very forward direction, within $3.5^{\circ}$ of the beam direction, is independent of $A$. These experiments are 'inclusive'; they study all final states, including the vast majority where the target nucleus has been broken up, in contrast to the unbroken nucleus involved in coherent production.

Tentative theoretical explanations by Gottfried (Phys. Rev. Lett., 32, 957; 1974), Goldhaber (Phys. Rev. Lett., 33, $47 ; 1974)$ and others have stressed the importance of the 'time dilation' effect of special relativity in understanding the passage of an excited lump of matter through a nucleus. At $200 \mathrm{GeV}$ it takes about $10^{-23}$ seconds for a particle to get out of a nucleus. But it may take ten times as long for a fast cluster of strongly interacting particles to get out of range of their mutual interaction. The coherent-production data show that the fast cluster behaves like one particle while it is still within the mutual interaction range. The data on inclusive multiplicities seem to be saying the same thing but with the added inference that the decay properties of the cluster are not modified by multiple collisions in nuclear matter. The results so far are fascinating, but there is scope for many more detailed measurements to be made. These could give a new source of evidence on the basic mechanisms of particle scattering, and on the structure of the particles.

\section{Changing views of mantle viscosity}

from Peter J. Smith

THE question of the Earth's internal viscosity first became prominent in connection with vertical movements of the crust, in particular with the process of isostatic readjustment. More recently, however, the problem has assumed a much greater significance because of the important part that mantle flow processes are thought to play in the large-scale horizontal motions of continental drift and seafloor spreading. The need to find a mechanism for such motions revived the popularity of the concept of the Earth as rigid lithosphere, viscous asthenosphere, rather more viscous mesosphere and fluid core (as opposed to the equally valid, but apparently less appropriate, division into crust, mantle and core). This emphasis on viscosity then led to much closer examination of the flow properties of the asthenosphere (particularly) and mesosphere, although there is still wide disagreement over the numerical values of viscosity.

In the early studies of isostatic recovery carried out by Haskell ( $A \mathrm{~m}$. J. Sci., 33, 22; 1937), Vening Meinesz (Proc. Kon. Ned. Akad. Wetensch., 40, $654 ; 1937)$ and others with reference to the uplift of Fennoscandia, it was assumed that the mantle flows as a
Newtonian fluid and has a uniform viscosity. This work led to the conclusion that, for an infinitely deep medium, the relaxation time (the time taken for the deviation from isostatic equilibrium to decrease to $1 / e$ of its initial value) is proportional to viscosity but inversely proportional to the linear dimension of the removed load. Unfortunately, when Crittenden ( $J$ geophys. Res., 68, 5517; 1963) came to analyse the uplift of Lake Bonneville, he found that the linear dimension differed from that of Fennoscandia by a factor of about 10 but that the relaxation times for the two areas were comparable. By this time, Takeuchi (J. geophys. Res., 68, 2357; 1963) had already offered a possible explanation of the discrepancy in his suggestion that flow may be concentrated in a thin layer in the upper mantle, basing his argument on the prior conclusion by Jeffreys (Geophys. J., 8, 196; 1952) that such layered flow would involve a quite different relationship between relaxation time and linear dimension. But this was not entirely satisfactory insofar as the isostatic processes in Lake Bonneville and Fennoscandia were only reconciled by the ad hoc assumption that the flow layers beneath the two areas differ in thickness.

As a result, McConnell (J. geophys. Res., 73, 7089; 1968) decided that the assumption of uniform viscosity must be discarded, and proposed instead a mantle model comprising layers of different viscosity, generally increasing with depth. In the mean time, Gordon (J. geophys. Res., 70, 2413; 1965) had obtained a broadly similar variation of viscosity with depth by considering flow in the mantle to be due to diffusion creep (Herring-Nabarro creep). Both models led to lower mantle viscosities comparable to the $10^{26}$ poise obtained by Macdonald (Space Sci. Rev., 2, 273; 1963) from his analysis of the response of the Earth's shape to the decrease in rotational velocity. Moreover, neither model gave any cause to suppose that the assumption of Newtonian flow need be rejected; on the contrary, in Gordon's model, Newtonian flow was apparently required by the fact that for diffusion creep, stress is proportional to strain (at least for small strains)

But this consistency and agreement was soon questioned by Weertman (Rev. Geophys. Space Phys., 8, 145; 1970) who concluded that whereas diffusion creep dominates at very low stresses (lower than about $10^{-2}$ bar), at higher stresses dislocation motion becomes important. Since dislocation motion involves a non-linear stressstrain relationship, the effect of Weertman's conclusion was to overthrow the idea of Newtonian flow in the mantle. His analysis also revealed 